\title{
Human Papillomavirus Prevalence and Type Distribution Among 968 Women in South Korea
}

\author{
Kyeong A So ${ }^{1}$, Jin Hwa Hong ${ }^{2}$, Jae Kwan Lee ${ }^{2}$ \\ 'Department of Obstetrics and Gynecology, Cheil General Hospital \& Women's Healthcare Center, Dankook University Medical College, ${ }^{2}$ Department \\ of Obstetrics and Gynecology, Korea University Guro Hospital, Korea University College of Medicine, Seoul, Korea
}

\begin{abstract}
Background: Geographic variation in the prevalence of carcinogenic types and human papillomavirus (HPV) genotype distribution is closely associated with the impact of HPV prophylactic vaccines. We determined the prevalence and distribution of HPV genotypes among healthy women in Korea.

Methods: This study included 968 healthy women who were examined at a health promotion center of the Korea University Guro Hospital between January and June 2013. Each participant had a Pap test and a HPV DNA test using the Anyplex ${ }^{\mathrm{TM}}$ || HPV 28 Detection system, which detects 19 high-risk HPVs (HR HPVs) and 9 low-risk HPVs (LR HPVs). Women with abnormal cytology and/or positivity for HR HPVs were referred to colposcopic biopsy.

Results: Overall HR HPV prevalence based on the assay was 33.7\%. Among them, 225 women had single infection and 101 women had multiple infection. The most frequently occurring HR HPV types were $53(6.5 \%), 52(6.1 \%), 58(4.8 \%), 16(4.5 \%)$, and $68(4.2 \%)$. The most frequently occurring LR HPV types were $54(5.4 \%), 70(3.8 \%), 42(3.6 \%), 61(3.4 \%)$, and $44(3.1 \%)$. The prevalence of HPV 16 was highest (17.6\%) among women with cervical intraepithelial neoplasia (CIN) and HPV 16 was strongly associated with a diagnosis of CIN2/3 (odds ratio $=20.5 ; 95 \%$ confidence interval: 3.9-107.1; $P<0.0001$ ).

Conclusions: HPV 53, 52, 58, 16, and 68 were common HR HPV types among healthy Korean women. HPV16 was the most common type in high-grade CIN lesions, as shown in most studies worldwide. The results might be useful information for cervical cancer prevention in South Korea.
\end{abstract}

(J Cancer Prev 2016;21:104-109)

Key Words: Human papillomavirus, Cervical intraepithelial neoplasia, Cytology

\section{INTRODUCTION}

Human papillomavirus (HPV) is one of the most common causes of sexually transmitted disease in women and results in various clinical conditions from asymptomatic infections to malignant genital diseases. More than 200 types of HPV have been identified on the basis of DNA sequence and approximately $30 \mathrm{HPV}$ types infect primarily the cervix, vagina, vulva, and anus. ${ }^{1}$ The relationship between HPV infection and cervical malignancy is well established. Based on their association with cervical cancer and precursor lesions, HPV types can also be grouped as high-risk (HR) or low-risk (LR): LR HPV includes types 6, 11, 40, 42, 43, and 44, whereas HR HPV includes types $16,18,31,33,35,39,45,51$, $52,56,58,59$, and 66 .

The overall prevalence of HPV infection worldwide is estimated to be approximately $10 \%(1.4 \%-25.6 \%)^{2}$. In contrast, in patients with cervical cancer, the prevalence of HPV DNA is as high as $99.7 \%$. $^{3}$ Therefore, detection of HR HPV is becoming increasingly attractive as a primary screening tool for cervical cancer because of its sensitivity and cost-effectiveness. ${ }^{4}$ However, the

Received March 5, 2016, Revised April 19, 2016, Accepted April 22, 2016

Correspondence to: Jae Kwan Lee

Department of Obstetrics and Gynecology, Korea University Guro Hospital, Korea University College of Medicine, 148 Gurodong-ro, Guro-gu, Seoul 08308, Korea

Tel: +82-2-2626-3142, Fax: +82-2-838-1560, E-mail: jklee38@korea.ac.kr, ORCID: Jae Kwan Lee, http://orcid.org/0000-0003-3101-6403

Copyright (C) 2016 Korean Society of Cancer Prevention

(c) This is an Open Access article distributed under the terms of the Creative Commons Attribution Non-Commercial License (http://creativecommons.org/licenses/by-nc/4.0) which permits unrestricted non-commercial use, distribution, and reproduction in any medium, provided the original work is properly cited. 
prevalence and distribution of HPV types differ around the world and the importance of different HPV types varies by region. In the United States and Europe, four types are most often found in patients with cervical cancer, with type 16 accounting for approximately $50 \%$ of cases, followed by types 18,31 , and $45 .{ }^{5}$ In South China, type 52 is the most common, followed by types 16 , 58,68 , and $33 .^{6}$

Knowledge of geographic variation in the prevalence of carcinogenic types and HPV genotype distribution is essential in order to assess the impact of HPV prophylactic vaccines. ${ }^{7}$ In South Korea, there are several reports on the HPV type distribution in cervical cancers but few studies on screening in healthy women. ${ }^{8-10}$ Therefore, we investigated the prevalence and distribution of HPV types among women who visited the health promotion center of Guro hospital and analyzed the risk factors associated with HPV infection.

\section{MATERIALS AND METHODS}

\section{Study population}

This study included 968 healthy women who were examined at a health promotion center of the Korea University Guro Hospital between January and June 2013. Each participant had a gynecologic examination that included liquid-based cytology and a cervical sample for HPV detection. All patient specimens and relevant data were obtained with approval from the Institutional Review Board of Korea University Guro Hospital and with informed patient consent.

\section{Liquid-based cytology}

A Cervex brush (Rovers Medical Devices, Oss, Netherlands) was used to obtain samples from the cervix. The brush was immediately rinsed in a vial of PreservCyt solution (Cytyc Corporation, Marlborough, MA, USA) and the vial was placed in a ThinPrep (Cytyc Corporation) processor. The ThinPrep slide was fixed in ethanol and stained with Papanicolaou's stain. Abnormal cytology was reported using the 2001 Bethesda system as atypical squamous cell of undetermined significance (ASCUS), low-grade squamous intraepithelial lesion (LSIL), or high-grade squamous intraepithelial lesion (HSIL).

\section{Human papillomavirus DNA testing}

HPV DNA testing was carried out on all samples using the Anyplex $^{\text {TM }}$ II HPV 28 Detection system (Seegene, Seoul, Korea). Extraction of total DNA was performed as recommended by the manufacturer. The kit contains sets of primers that were specifically designed based on highly conserved regions of the HPV genetic sequences and uses HPV-specific dual priming oligonucleotides $\left(\mathrm{DPO}^{\mathrm{TM}}\right)$. This multiplex real-time $\mathrm{PCR}$ test identifies $28 \mathrm{HPV}$ genotypes including $19 \mathrm{HR}$ HPVs $(16,18,26,31$, $33,35,39,45,51,52,53,56,58,59,66,68,69,73,82)$ and 9 LR HPVs $(6,11,40,42,43,44,54,61,70)$. Data recording and interpretation were automated with the Anyplex ${ }^{\mathrm{TM}}$ software according to the manufacturer's instructions.

\section{Histology}

Women with abnormal cytology and/or HR HPV positivity were referred to colposcopic examination and cervical biopsy. All biopsy specimens submitted for histologic assessment were routinely examined in their entirety. Histologic grading of cervical intraepithelial neoplasia (CIN) is based on the standard grades of CIN1, CIN2, and CIN3.

\section{Statistical analysis}

Data are presented as the mean \pm SD for continuous variable and number (percentage) for categorical variables. The prevalence of HPV was analyzed with respect to cytology results based on the Bethesda system. When two groups compared, differences in continuous variable were analyzed using the Student's $t$-test and proportions were compared using the chi-square test. The Mann-Whitney U-test was used to assess the association between HPV status and parity and abortion. To estimate the risk of high-grade CIN (CIN2/3) associated with different HPV types, we calculated odds ratios (ORs) and 95\% confidence intervals (CIs) by logistic regression. The ORs were estimated for common five types of HPV among the HR HPV infected CIN cases. Values of $P<$ 0.05 were considered to indicate a statistically significant result. Data were analyzed with IBM SPSS ver. 20.0 (IBM Co., Armonk, NY, USA).

\section{RESULTS}

A total of 968 healthy women were enrolled in this study. Table 1 presents the characteristics of the participants. The mean age of the study population was 48.3 years. With respect to cervical cytology, 610 women (63.0\%) had normal cytology, 112 (11.6\%) had ASCUS, 67 women (6.9\%) had LSIL, and 15 women (1.6\%) had HSIL. HPV type testing by the Anyplex ${ }^{\mathrm{TM}}$ II HPV 28 Detection system revealed that 393 women (40.6\%) were positive for HPV (199 women for only HR HPV infection, 67 women for only LR HPV infection, and 127 women for both HR and LR HPV infections). For HR HPV infection, HPV 53 (6.5\%) was the most 
Table 1. Baseline characteristics of women in this study $(n=968)$

\begin{tabular}{|c|c|}
\hline Characteristic & Value \\
\hline Age (yr) & $48.3 \pm 11.7$ \\
\hline$<30$ & $61(6.3)$ \\
\hline $30-39$ & $166(17.1)$ \\
\hline $40-49$ & $275(28.4)$ \\
\hline $50-59$ & $313(32.3)$ \\
\hline$\geq 60$ & $153(15.8)$ \\
\hline Parity & $1.8 \pm 1.2$ \\
\hline Body mass index $\left(\mathrm{kg} / \mathrm{m}^{2}\right)$ & $23.5 \pm 3.8$ \\
\hline \multicolumn{2}{|l|}{ Cervical cytology } \\
\hline Normal & $610(63.0)$ \\
\hline ASCUS & $112(11.6)$ \\
\hline LSIL & $67(6.9)$ \\
\hline HSIL & $15(1.5)$ \\
\hline Missing data & $164(17.0)$ \\
\hline \multicolumn{2}{|l|}{ HPV infection } \\
\hline Negative & $575(59.4)$ \\
\hline Positive & $393(40.6)$ \\
\hline High-risk only & $199(50.7)$ \\
\hline Low-risk only & $67(17.0)$ \\
\hline High- and low-risk & $127(32.3)$ \\
\hline \multicolumn{2}{|l|}{ HPV type specific prevalence } \\
\hline \multicolumn{2}{|l|}{ High-risk type } \\
\hline HPV 53 & $63 / 968(6.5)$ \\
\hline HPV 52 & $59 / 968(6.1)$ \\
\hline HPV 58 & $46 / 968(4.8)$ \\
\hline HPV 16 & $44 / 968(4.5)$ \\
\hline HPV 68 & $41 / 968(4.2)$ \\
\hline HPV 51 & $34 / 968(3.5)$ \\
\hline HPV 39 & $24 / 968(2.5)$ \\
\hline HPV 56 & $24 / 968(2.5)$ \\
\hline HPV 35 & $20 / 968(2.1)$ \\
\hline HPV 66 & $20 / 968(2.1)$ \\
\hline HPV 18 & $17 / 968(1.8)$ \\
\hline HPV 82 & $10 / 968(1.0)$ \\
\hline HPV 45 & $6 / 968(0.6)$ \\
\hline \multicolumn{2}{|l|}{ Low-risk type } \\
\hline HPV 54 & $52 / 968(5.4)$ \\
\hline HPV 70 & $37 / 968(3.8)$ \\
\hline HPV 42 & $35 / 968(3.6)$ \\
\hline HPV 61 & $33 / 968 \quad(3.4)$ \\
\hline HPV 44 & $30 / 968 \quad(3.1)$ \\
\hline HPV 43 & $27 / 968 \quad(2.8)$ \\
\hline HPV 40 & $20 / 968(2.1)$ \\
\hline HPV 6 & $10 / 968(1.0)$ \\
\hline HPV 11 & $3 / 968 \quad(0.3)$ \\
\hline
\end{tabular}

Values are presented as mean $\pm \mathrm{SD}$, number (\%), or number/total number (\%). ASCUS, atypical squamous intraepithelial lesion; LSIL, low-grade squamous intraepithelial lesion; HSIL, high-grade squamous intraepithelial lesion; HPV, human papillomavirus.

common type, followed by HPV 52 (6.1\%), HPV 58 (4.8\%), HPV 16 (4.5\%), HPV 68 (4.2\%), HPV51 (3.5\%), HPV 39 (2.5\%), HPV 56 (2.5\%), HPV 35 (2.1\%), HPV 66 (2.1\%), HPV 18 (1.8\%), HPV 82 (1.0\%), and HPV 45 (0.6\%). For LR HPV infection, HPV 54 (5.4\%) was the most
Table 2. Distribution of high-risk $\mathrm{HPV}^{\mathrm{a}}$ infections

\begin{tabular}{crl}
\hline & Variable & High-risk HPV/ \\
total HPV & infection \\
\hline Single infection & $225 / 393$ & $(57.3)$ \\
HPV 52 & $31 / 225$ & $(13.8)$ \\
HPV 58 & $27 / 225$ & $(12.0)$ \\
HPV 53 & $26 / 225$ & $(11.6)$ \\
HPV 16 & $25 / 225$ & $(11.1)$ \\
HPV 68 & $21 / 225$ & $(9.3)$ \\
HPV 51 & $15 / 225$ & $(6.7)$ \\
HPV 18 & $12 / 225$ & $(5.3)$ \\
HPV 56 & $12 / 225$ & $(5.3)$ \\
HPV 66 & $12 / 225$ & $(5.3)$ \\
HPV 39 & $9 / 225$ & $(4.0)$ \\
HPV 33 & $8 / 225$ & $(3.6)$ \\
HPV 35 & $8 / 225$ & $(3.6)$ \\
HPV 59 & $8 / 225$ & $(3.6)$ \\
HPV 31 & $5 / 225$ & $(2.2)$ \\
HPV 82 & $4 / 225$ & $(1.8)$ \\
HPV 45 & $1 / 225$ & $(0.4)$ \\
HPV 69 & $1 / 225$ & $(0.4)$ \\
Multiple infection & $101 / 393$ & $(25.7)$ \\
2 types & $76 / 101$ & $(75.2)$ \\
3 types & $22 / 101$ & $(21.8)$ \\
4 types & $2 / 101$ & $(2.0)$ \\
5 types & $1 / 101$ & $(1.0)$ \\
Total high-risk HPV infection & $326 / 393$ & $(83.0)$ \\
\hline
\end{tabular}

Values are presented as number/total number (\%). HPV, human papillomavirus. ${ }^{a} \mathrm{HPV} 16,18,26,31,33,35,39,45,51,52,53,56,58$, $59,66,68,69,73$, and 82 .

common type, followed by HPV 70 (3.8\%), HPV 42 (3.6\%), HPV 61 (3.4\%), HPV 44 (3.1\%), HPV 43 (2.8\%), HPV 40 (2.1\%), HPV 6 (1.0\%), and HPV $11(0.3 \%)$

The distributions of single and multiple HR HPV infection were shown in Table 2. Infection with a single HPV type and multiple HPV types was detected in $57.3 \%$ and $25.7 \%$ of all HPV-positive women, respectively. Among cases of HR HPV single infection, HPV 52 was the most frequent type (13.8\%), followed by HPV 58 (12.0\%), HPV 53 (11.6\%), HPV 16 (11.1\%), HPV 68 (9.3\%), HPV 51 (6.7\%), HPV 18, 56, and 66 (each 5.3\%), HPV 39 (4.0\%), HPV 33. 35, and 59 (each 3.6\%), HPV 31(2.2\%), HPV 82 (1.8\%), and HPV 45 and 69 (each 0.4\%).

To investigate risk factors associated with HR HPV infection, we compared several variables between HR HPV-positive and HPV-negative groups. As shown in Table 3, women with HR HPV had a significantly younger age at the first delivery (25.0 years versus 26.0 years, $P=0.006$ ) than the HPV-negative group. Other factors, including age, body mass index, the number of abortions or deliveries, HPV vaccination, menopause status, underlying diseases (diabetes, hypertension), and condom use were not 
significantly different between the two groups.

HPV prevalence rates according to cervical cytology are shown in Figure 1. The prevalence of HR HPV was $26.9 \%$ in patients with normal cytology, $45.6 \%$ for ASCUS, 79.1\% for LSIL, and $93.3 \%$ for HSIL. The prevalence of HR HPV increased significantly with increasing grade from normal to HSIL $(P<0.001)$. Women with abnormal cytology and/or HR HPV positivity $(n=73)$ were referred for colposcopic examination and cervical biopsy (Table 4). Among these, 55 women had CIN and only 18 had normal histologic

Table 3. Characteristics of women infected with high-risk HPVs ${ }^{a}$

\begin{tabular}{lcccc}
\hline \multicolumn{1}{c}{ Characteristic } & $\begin{array}{c}\text { HPV negative } \\
(\mathrm{n}=575)\end{array}$ & $\begin{array}{c}\text { HPV positive } \\
(\mathrm{n}=326)\end{array}$ & $P$-value \\
\hline Age (yr) & $48.7 \pm 11.1$ & $47.5 \pm 12.4$ & 0.148 \\
Body mass index $\left(\mathrm{kg} / \mathrm{m}^{2}\right)$ & $23.5 \pm 3.8$ & $23.5 \pm 3.8$ & 0.846 \\
Abortions & $1.0(0-22)$ & $1.0(0-8)$ & 0.458 \\
Deliveries & $2.0(0-7)$ & $2.0(0-8)$ & 1.000 \\
Age at first delivery $(\mathrm{yr})$ & $26.0 \pm 4.4$ & $25.0 \pm 5.3$ & 0.006 \\
HPV vaccination & $56(9.7)$ & $28(8.6)$ & 1.000 \\
Menopause & $219(38.1)$ & $102(31.3)$ & 0.423 \\
Diabetes & $22(3.8)$ & $7(2.1)$ & 0.322 \\
Hypertension & $72(12.5)$ & $43(13.2)$ & 0.400 \\
Contraception & & & 0.110 \\
$\quad$ Condom & $42(7.3)$ & $24(7.4)$ & \\
$\quad$ Others & & $122(21.2)$ & $89(27.3)$ & \\
$\quad$ None & $411(71.5)$ & $213(65.3)$ & \\
\hline
\end{tabular}

Values are presented as mean $\pm \mathrm{SD}$, median (range), or number (\%). HPV, human papillomavirus. ${ }^{\mathrm{a}} \mathrm{HPV} 16,18,26,31,33,35,39,45$, $51,52,53,56,58,59,66,68,69,73,82$. 'Oral pill use, intrauterine device, vasectomy, bilateral salpingectomy.

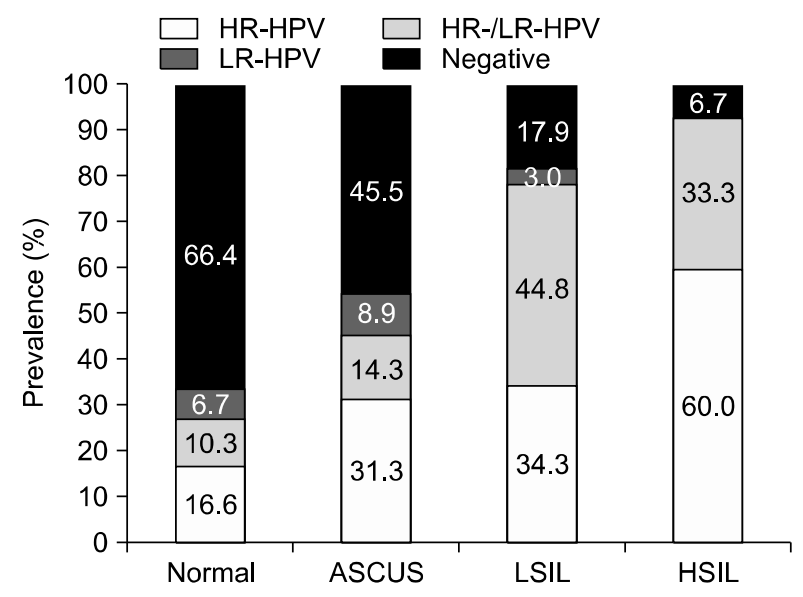

Figure 1. Prevalence of HPV infection according to cervical cytology. The prevalence of HR HPVs increases significantly with increasing grade from normal to HSIL $(P<0.001)$. ASCUS, atypical squamous intraepithelial lesion; LSIL, low-grade squamous intraepithelial lesion; HSIL, high-grade squamous intraepithelial lesion; HPV, human papillomavirus; HR-HPV, high-risk HPV; LR-HPV, low-risk HPV; HR-/LR-HPV, high-risk and low-risk HPV co-infection. findings. The HR HPV positivity rate was $44.4 \%$ in the normal group, $86.5 \%$ in CIN1, $100 \%$ in CIN2, and $92.9 \%$ in CIN3, whereas the percentage of patients with abnormal cytology was $66.7 \%$ in normal group, $81.1 \%$ in CIN1, $75.0 \%$ in CIN2, and $78.6 \%$ in CIN3. These results suggest that HR HPV infection was more predictive for cervical lesions than abnormal cytology. In addition we evaluated the types of HR HPV most commonly involved in single infection in the patients with CIN. HPV 16 (17.6\%) was most common type in patients with CIN, followed by HPV 58 (13.2\%), HPV 52 (8.8\%), HPV 39 (7.4\%), and HPV 51 (7.4\%). Of these, HPV 16 showed the strongest association with a diagnosis of CIN2/3 (OR $=20.5$; 95\% CI: 3.9-107.1; $P<0.0001$ ) (Table 5).

\section{DISCUSSION}

This cross-sectional study provided the type-specific prevalence of HPV in healthy Korean women through testing for $28 \mathrm{HPV}$ types. The overall prevalence of HPV infection was $40.6 \%$ ( $n=$

Table 4. Results of colposcopic biopsy

\begin{tabular}{lcrr}
\hline \multicolumn{1}{c}{ Result } & Case & $\begin{array}{c}\text { Abnormal } \\
\text { cytology }\end{array}$ & $\begin{array}{r}\text { Positive for } \\
\text { high-risk HPV }\end{array}$ \\
\hline Normal & $18(24.7)$ & $12 / 18(66.7)$ & $8 / 18(44.4)$ \\
CIN1 & $37(50.7)$ & $30 / 37(81.1)$ & $32 / 37(86.5)$ \\
CIN2 & $4(5.5)$ & $3 / 4(75.0)$ & $4 / 4(100)$ \\
CIN3 & $14(19.2)$ & $11 / 14(78.6)$ & $13 / 14(92.9)$ \\
Total & $73(100)$ & & \\
\hline
\end{tabular}

Values are presented as number (\%). HPV, human papillomavirus; CIN, cervical intraepithelial neoplasia.

Table 5. HPV type-specific risk for HSIL (CIN2/3) in HR HPV ${ }^{\mathrm{a}}$ infected women

\begin{tabular}{cccc}
\hline HPV type & $\begin{array}{c}\text { HSIL/ } \\
\text { total CIN }\end{array}$ & $\begin{array}{c}\text { Odds ratio } \\
(95 \% \mathrm{CI})\end{array}$ & $P$-value \\
\hline HPV 16 & $10 / 12$ & $20.5(3.9-107.1)$ & $<0.0001$ \\
Other HR HPVs & $11 / 56$ & & \\
HPV 58 & $1 / 9$ & $0.2(0.0-2.1)$ & 0.256 \\
Other HR HPVs & $20 / 59$ & & \\
HPV 52 & $2 / 6$ & $1.1(0.2-6.1)$ & 1.000 \\
Other HR HPVs & $19 / 62$ & & \\
HPV 51 & $1 / 5$ & $0.5(0.1-5.1)$ & 1.000 \\
Other HR HPVs & $20 / 63$ & & \\
HPV 39 & $2 / 5$ & $1.5(0.2-10.0)$ & 0.641 \\
Other HR HPVs & $19 / 63$ & & \\
\hline
\end{tabular}

HPV, human papillomavirus; HSIL, high-grade intraepithelial lesion; CIN, cervical intraepithelial neoplasia; HR, high-risk; CI, confidence interval. ${ }^{a} \mathrm{HPV} 16,18,26,31,33,35,39,45,51,52,53,56,58,59$, $66,68,69,73,82$. 
393). Among the HPV-positive group, 326 women (83.0\%) were infected with at least one HR type and only 67 women (17.0\%) were infected with a LR type. The results of this study indicate a much higher prevalence of HPV than the overall prevalence worldwide (1.4\%-25.6\%). The prevalence of HR HPV was also high (33.7\%) and it is unusual in the advanced age women. The result may be caused by specific study population who visited the health promotion center. They are different group from the general healthy population.

The most common types are HPV 53 (6.5\%), HPV 52 (6.1\%), HPV 58 (4.8\%), HPV 16 (4.5\%), and HPV 68 (4.2\%). In previous Korean studies, HPV 52, HPV 58, and HPV 51 were commonly identified in precancerous lesions, whereas HPV 16, HPV 33, HPV 58, and HPV 31 were the most common types in cervical cancer. ${ }^{8-10}$ These results for HR HPV genotyping are slightly different from those obtained in another Korean study which was conducted in screening population of Gangnam. ${ }^{11}$ Its overall prevalence of HR HPV was $19.7 \%$, with the most common types being HPV 58, HPV 16, HPV 52, and HPV 18. Differences in the prevalence of HPV genotypes might be related to the complex geographical factors and biological interactions between different HPV types and host immune system. ${ }^{12}$ The Gangnam population in South of Seoul consists of people with higher socioeconomic status than those in Guro, West of Seoul. A high education level is a possible protective factor for HPV infection. ${ }^{13}$ In addition, women from Gangnam may seek health check-ups more frequently and thus have a substantially lower prevalence of abnormal cervical cytology than those from Guro (less than 5\% versus 20\%).

Among the HPV-positive group, 57.3\% had HR HPV single infection and $25.7 \%$ had co-infection with multiple HR HPVs. Multiple infection with different HR HPV types was more common in this study than in a previous Korean study using a HPV DNA chip (157/967, 16.2\%). ${ }^{10}$ This difference might be related to use of the Anyplex ${ }^{\mathrm{TM}}$ II HPV 28 assay that allowed detection of nearly all genital genotypes (19 HR HPV types) compared with previous HPV DNA chip analysis, which measured only 15 HR HPV types. The Anyplex ${ }^{\mathrm{TM}}$ II HPV28 assay is a very sensitive and specific HPV genotyping assay. ${ }^{14}$ It is more sensitive in the detection of HR types represented by Hybrid Capture 2 assay and exhibits a higher concordance with comprehensive genotyping based on the sequencing analysis. ${ }^{15}$ Depending on the HPV detection method used, multiple infection rates of up to $40 \%$ have been reported. ${ }^{16}$ In addition, high rate of multiple HR HPV infections might result from the deviated study population who had the high prevalence (33.7\%) of HR HPV infection. Study population, that is, women who visited health promotion center, also can be a different group from the general healthy population.

Multiple HPV infection has been observed more frequently in women with abnormal cytology or those with impaired immune system. ${ }^{17-19}$ In addition, multiple HPV infection seems to promote cervical carcinogenesis, increasing the risk of high-grade intraepithelial lesions and invasive cancer through a synergistic effect of HPV types. ${ }^{20,21}$ Therefore, the relatively high rate of multiple HR HPV infection in Korean women has implications for the screening of cervical cancer and prediction of the outcome of HPV infections.

Understanding the HPV prevalence and type distribution in women is important for determining the effectiveness of prophylactic HPV vaccination and HPV-based cervical cancer screening. Women infected with a HR HPV type or with multiple HPV types have an increased risk for persistent $\mathrm{CIN}^{22}$ In this study, the overall prevalence of HR HPV among women with normal cytology was $26.9 \%$. This was higher than the previously reported worldwide distribution (13.3\%) but similar to results of a Korean meta-analysis (23.9\%). ${ }^{2.9}$ In addition, the incidence of ASCUS is relatively high as $11.6 \%$ may result from high prevalence of HR HPV infection around $33.7 \%$.

The prevalence significantly increased according to the cytologic grade from ASCUS to HSIL (45.6\% in ASCUS, $79.1 \%$ in LSIL, and 93.3\% in HSIL, $P<0.001$ ). HPV prevalence is also positively associated with the severity of cervical abnormalities. ${ }^{6}$ Recently, a report on 30,371 women in China showed that the HR HPV prevalence was $11.2 \%$ in women with normal histology, $79.7 \%$ in CIN1, 95.0\% in CIN2, 97.4\% in CIN3, and 98.0\% in carcinoma. ${ }^{23}$ In this study the HR HPV prevalence was $44.4 \%$ in women with normal histology, $86.5 \%$ in CIN1, $100 \%$ in CIN2, and $92.4 \%$ in CIN3. In addition, this relationship seemed to be more predictive of cervical lesions than abnormal cytology $(66.7 \%$ in normal group, $81.1 \%$ in CIN1, $75.0 \%$ in CIN2, and $78.6 \%$ in CIN3). HPV testing for a group of HR types has previously been shown to be a more sensitive screening technique than cytology for detecting high-grade CIN ${ }^{4}$ Among the CIN lesions, HPV 16 (17.6\%) was the most common type in patients with CIN and the most strongly associated with a diagnosis of CIN2/3 (OR $=20.5 ; 95 \% \mathrm{CI}$ : 3.9-107.1; $\quad P<0.0001$ ), as demonstrated in most studies worldwide.

This study has some limitations. First, there can be selection bias. The study population was relatively small and in relatively advanced age group (mean age: 48.3 years old) visited the health promotion center. The results should be interpreted with caution, especially since the participants may differ from the general population. Second, a single institution has geographical limita- 
tion and current estimation of HR-HPV prevalence is not equivalent to a national average. Nation-wide study is needed to confirm accurate prevalence of HPV infection.

Nonetheless, these findings can provide useful basic data on the status of HPV infection in Korea, especially since there is histological confirmation of the lesions, and attribute the lesion to the specific HPV type. In conclusion, the most common HR HPV types were types 53 (6.5\%), 52 (6.1\%), 58 (4.8\%), $16(4.5 \%)$, and 68 (4.2\%). Also, this study confirms the clinical importance of HPV type 16 , the most common type in high-grade CIN lesions. Information on regional HPV prevalence might be useful for establishment of a strategy for cervical cancer prevention in South Korea. Further large-scale and multicenter studies will be needed to fully understand the HPV status among Korean women.

\section{CONFLICTS OF INTEREST}

No potential conflicts of interest were disclosed.

\section{REFERENCES}

1. Burd EM. Human papillomavirus and cervical cancer. Clin Microbiol Rev 2003;16:1-17.

2. Clifford GM, Gallus S, Herrero R, Muñoz N, Snijders PJ, Vaccarella S, et al; IARC HPV Prevalence Surveys Study Group. Worldwide distribution of human papillomavirus types in cytologically normal women in the International Agency for Research on Cancer HPV prevalence surveys: a pooled analysis. Lancet 2005;366:991-8.

3. Walboomers JM, Jacobs MV, Manos MM, Bosch FX, Kummer JA, Shah KV, et al. Human papillomavirus is a necessary cause of invasive cervical cancer worldwide. J Pathol 1999;189:12-9.

4. Cuzick J, Szarewski A, Cubie H, Hulman G, Kitchener H, Luesley $D$, et al. Management of women who test positive for high-risk types of human papillomavirus: the HART study. Lancet 2003;362:1871-6.

5. Harro CD, Pang YY, Roden RB, Hildesheim A, Wang Z, Reynolds MJ, et al. Safety and immunogenicity trial in adult volunteers of a human papillomavirus $16 \mathrm{~L} 1$ virus-like particle vaccine. J Natl Cancer Inst 2001;93:284-92.

6. Li J, Huang R, Schmidt JE, Qiao YL. Epidemiological features of Human Papillomavirus (HPV) infection among women living in Mainland China. Asian Pac J Cancer Prev 2013;14:4015-23.

7. Muñoz N, Bosch FX, de Sanjosé S, Herrero R, Castellsagué X, Shah KV, et al; International Agency for Research on Cancer Multicenter Cervical Cancer Study Group. Epidemiologic classification of human papillomavirus types associated with cervical cancer. N Engl J Med 2003;348:518-27.

8. Oh JK, Alemany L, Suh JI, Rha SH, Muñoz N, Bosch FX, et al. Type-specific human papillomavirus distribution in invasive cervical cancer in Korea, 1958-2004. Asian Pac J Cancer Prev 2010;11:993-1000.
9. Bae JH, Lee SJ, Kim CJ, Hur SY, Park YG, Lee WC, et al. Human papillomavirus (HPV) type distribution in Korean women: a meta-analysis. J Microbiol Biotechnol 2008; 18:788-94.

10. Lee HS, Kim KM, Kim SM, Choi YD, Nam JH, Park CS, et al. Human papillomavirus genotyping using HPV DNA chip analysis in Korean women. Int J Gynecol Cancer 2007;17:497-501.

11. Kim MJ, Kim JJ, Kim S. Type-specific prevalence of high-risk human papillomavirus by cervical cytology and age: data from the health check-ups of 7,014 Korean women. Obstet Gynecol Sci 2013:56:110-20.

12. Hildesheim A, Wang SS. Host and viral genetics and risk of cervical cancer: a review. Virus Res 2002;89:229-40.

13. Chen Z, Meng W, Du R, Zhu Y, Zhang Y, Ding Y. Genotype distribution and the relative risk factors for human papillomavirus in Urumqi, China. Exp Ther Med 2013;6:85-90.

14. Estrade C, Sahli R. Comparison of Seegene Anyplex II HPV28 with the PGMY-CHUV assay for human papillomavirus genotyping. J Clin Microbiol 2014:52:607-12.

15. Kwon MJ, Roh KH, Park H, Woo HY. Comparison of the Anyplex II HPV28 assay with the Hybrid Capture 2 assay for the detection of HPV infection. J Clin Virol 2014:59:246-9.

16. Hamlin-Douglas LK, Coutlée F, Roger M, Franco EL, Brassard P. Prevalence and age distribution of human papillomavirus infection in a population of Inuit women in Nunavik, Quebec. Cancer Epidemiol Biomarkers Prev 2008;17:3141-9.

17. Ho GY, Palan PR, Basu J, Romney SL, Kadish AS, Mikhail M, et al. Viral characteristics of human papillomavirus infection and antioxidant levels as risk factors for cervical dysplasia. Int J Cancer 1998; 78:594-9.

18. Levi JE, Kleter B, Quint WG, Fink MC, Canto CL, Matsubara R, et al. High prevalence of human papillomavirus (HPV) infections and high frequency of multiple HPV genotypes in human immunodeficiency virus-infected women in Brazil. J Clin Microbiol 2002; 40:3341-5.

19. Rousseau MC, Abrahamowicz M, Villa LL, Costa MC, Rohan TE, Franco EL. Predictors of cervical coinfection with multiple human papillomavirus types. Cancer Epidemiol Biomarkers Prev 2003:12:1029-37.

20. van der Graaf Y, Molijn A, Doornewaard H, Quint W, van Doorn LJ, van den Tweel J. Human papillomavirus and the long-term risk of cervical neoplasia. Am J Epidemiol 2002;156:158-64.

21. Trottier H, Mahmud S, Costa MC, Sobrinho JP, Duarte-Franco E, Rohan TE, et al. Human papillomavirus infections with multiple types and risk of cervical neoplasia. Cancer Epidemiol Biomarkers Prev 2006;15:1274-80.

22. Ho GY, Einstein MH, Romney SL, Kadish AS, Abadi M, Mikhail M, et al; Albert Einstein Cervix Dysplasia Clinical Consortium. Risk factors for persistent cervical intraepithelial neoplasia grades 1 and 2: managed by watchful waiting. J Low Genit Tract Dis 2011;15:268-75.

23. Zhao FH, Lin MJ, Chen F, Hu SY, Zhang R, Belinson JL, et al; Cervical Cancer Screening Group in China. Performance of high-risk human papillomavirus DNA testing as a primary screen for cervical cancer: a pooled analysis of individual patient data from 17 population-based studies from China. Lancet Oncol 2010;11:1160-71. 